\title{
Le trouble développemental du langage (TDL) : mise à jour interdisciplinaire
}

\author{
Chantale Breault ${ }^{1,2,4}$, M. P. O., Marie-Julie Béliveau ${ }^{2,3}$, Ph. D., Fannie Labelle ${ }^{2,3}$, B. Sc., \\ Florence Valade $^{2,3}$, B. Sc. et Natacha Trudeau ${ }^{1,4,5}, \mathrm{Ph}$. D. \\ ${ }^{1}$ École d'orthophonie et d'audiologie, Université de Montréal \\ ${ }^{2}$ Centre de recherche du CIUSSS Nord-de-l'Île-de-Montréal \\ ${ }^{3}$ Département de psychologie, Université de Montréal \\ ${ }^{4}$ Centre de recherche interdisciplinaire en réadaptation du Montréal métropolitain \\ ${ }^{5}$ Centre de recherche du CHU Sainte-Justine
}

\begin{abstract}
Bien que le trouble développemental du langage (TDL) soit fréquent (7,58 \%; Norbury et al., 2016) et ait des impacts perdurant jusqu'à l'âge adulte (Feeney, Desha, Khan, Ziviani, \& Nicholson, 2016), il est beaucoup moins connu que d'autres problématiques telles que le trouble du déficit de l'attention avec ou sans hyperactivité (TDAH) ou le trouble du spectre de l'autisme (TSA). L'inconstance des définitions selon les domaines (p. ex., médecine, éducation, psychologie, orthophonie) pourrait partiellement expliquer cette méconnaissance (Bishop, 2017). Depuis peu, une terminologie et une démarche menant au diagnostic de TDL font l'objet d'un consensus international multidisciplinaire, et ce, grâce au projet CATALISE (Bishop, Snowling, Thompson, Greenhalgh, \& CATALISE-consortium, 2016, 2017). Le but de cet article est de présenter une mise à jour des enjeux et des connaissances actuelles liés au TDL en s'intéressant aux changements d'appellation et de critères initiés par le projet CATALISE. Pour les professionnels et chercheurs œuvrant dans le domaine, il s'agit d'une occasion de réfléchir aux besoins des personnes vivant avec un TDL.
\end{abstract}

Mots clés : trouble développemental du langage, évaluation, interdisciplinarité, terminologie, enfant

\begin{abstract}
Although developmental language disorder (DLD) is common (7.58\%; Norbury et al., 2016) and has impacts lasting into adulthood (Feeney, Desha, Khan, Ziviani, \& Nicholson, 2016), it is not as renowned than other disorders as attention deficit hyperactivity disorder (ADHD) or autism spectrum disorder (ASD). Inconsistent definitions across domains (e.g., medicine, education, psychology, speech-language pathology) may partially explain this situation (Bishop, 2017). Recently, a terminology and an approach leading to the diagnosis of DLD were the subject of a multidisciplinary international consensus, due to the CATALISE project (Bishop, Snowling, Thompson, Greenhalgh \& CATALISE-consortium, 2016, 2017). The purpose of this paper is to present an update of current issues and knowledge related to DLD by looking at the criteria and terminology changes initiated by the CATALISE project. For professionals and researchers working in the field, this is an opportunity to reflect on the needs of people living with DLD.
\end{abstract}

Keywords: developmental language disorder, assessment, interdisciplinary, terminology, children

Une pratique fondée sur les données probantes exige une constante mise à jour des connaissances. Le défi est grand dans les domaines impliquant une collaboration interprofessionnelle, puisque la littérature scientifique et les balises cliniques ne circulent pas toujours d'une profession à l'autre. C'est particulièrement vrai en ce qui concerne le trouble développemental du langage (TDL), dont la conceptualisation chevauche plusieurs disciplines, celles-ci l'abordant chacune avec des points de vue et des terminologies différentes. Parfois appelé « dysphasie » ou « trouble primaire du langage », le

La correspondance concernant cet article doit être adressée à / Correspondence concerning this article should be addressed to: Chantale Breault, Université de Montréal,

Courriel/e-mail : chantalebreault@hotmail.com
TDL n'a pas de cause connue. Il peut affecter différentes composantes $\mathrm{du}$ langage, incluant l'expression ou la compréhension des sons, des phrases ou du discours, le vocabulaire et l'accès lexical, le traitement et la mémoire verbale, ainsi que la pragmatique et l'ajustement au contexte de communication. Les difficultés apparaissent généralement en bas âge, persistent à long terme et ont des conséquences sur la vie quotidienne, sociale et scolaire des personnes atteintes. Le TDL a également des impacts en modalité écrite sur les habiletés à comprendre et à s'exprimer par la lecture et l'écriture.

Il existe un manque de consensus dans la définition du TDL chez les divers professionnels, et ce, même à l'intérieur des disciplines concernées. Par le projet 
CATALISE, un regroupement international et interdisciplinaire de 57 experts (Bishop et al., 2017) s'est récemment attaqué à cette problématique et a proposé d'adopter une appellation ainsi que des critères communs associés au TDL. L'Ordre des orthophonistes et audiologistes du Québec (OOAQ) a officiellement adhéré à ce consensus en septembre 2017, suivi par Orthophonie et Audiologie Canada (OAC) en décembre 2018. Dans sa revue professionnelle, l'American Speech-LanguageHearing Association (ASHA) a plutôt choisi de présenter les recommandations de CATALISE sous forme de débat (Volkers, 2018). Le but du présent article est de s'intéresser à la nature et aux implications des changements proposés, en y ajoutant une mise à jour utile aux professionnels et aux chercheurs œuvrant dans le domaine en contexte québécois. Pour ce faire, la problématique, le survol et l'analyse de certaines recommandations du consortium CATALISE, les défis d'arrimage de la nouvelle terminologie ainsi que les retombées à prévoir seront abordées.

\section{Problématique}

Les études internationales établissent la prévalence des troubles développementaux du langage (TDL) chez l'enfant à environ $7 \%$, avec une certaine variabilité dépendant de l'âge ou de la définition employée (McLeod \& Harrison, 2009; Tomblin et al., 1997). Selon une étude populationnelle récente, 7,58 $\%$ des enfants de quatre à cinq ans ont un trouble du langage d'origine inconnue, auxquels s'ajoute 2,34\% d'enfants ayant un trouble du langage associé à une déficience intellectuelle ou à une autre condition médicale identifiée (p. ex., trouble auditif ou visuel, paralysie cérébrale, épilepsie, trouble du spectre de l'autisme [TSA], syndrome de Down, neurofibromatose; Norbury et al., 2016).

La prévalence du TDL a longtemps été difficile à établir en raison de problèmes méthodologiques et de manque de constance dans la terminologie. Les problèmes méthodologiques les plus fréquents sont la variabilité et la subjectivité des critères menant à l'inclusion ou à l'exclusion des participants selon les études. Par exemple, plusieurs auteurs ont uniquement considéré les enfants dont le QI non verbal était supérieur à 85 , alors que des recherches ont ensuite démontré que cette mesure n'était pas stable dans le temps (Botting, 2005) ou différait selon les tests employés (Miller \& Gilbert, 2008). D'autres chercheurs ont établi que les résultats à un ou plusieurs tests langagiers devaient être inférieurs à un seuil arbitraire (p. ex., -2 ou -1,25 écarts-types), mais la variabilité des tests employés et le manque de considération de la sensibilité et de la spécificité de ces outils ont été critiqués (Spaulding, Plante, \&
Farinella, 2006). Il y a près de quarante ans, un article identifiait déjà ces enjeux en relevant que la prévalence de difficultés de langage chez l'enfant de trois ans variait de $0,07 \%$ à $5,3 \%$ (Silva, 1980). Cette prévalence variait selon la définition utilisée.

Déjà à cette époque, il était également relevé que l'inconstance de la prévalence calculée pouvait entraver l'offre de services, alors que des difficultés langagières non prises en charge causaient à certains enfants des " séquelles émotionnelles, sociales et éducatives » significatives (Rutter \& Martin, 1972, cité dans Silva, 1980). En effet, plusieurs études ont documenté que le TDL est associé à des impacts négatifs et persistants à l'enfance, l'adolescence et l'âge adulte dans des domaines aussi variés que la réussite scolaire, la socialisation, la santé mentale, l'accès à l'emploi et à l'autonomie (Conti-Ramsden \& Botting, 2008; Elbro, Dalby, \& Maarbjerg, 2011; St Clair, Pickles, Durkin, \& Conti-Ramsden, 2011). Bien que les mécanismes expliquant ces associations soient encore mal compris, des études longitudinales tendent à démontrer que ce ne sont pas nécessairement les habiletés langagières qui expliquent, en soi, les conséquences négatives vécues par les enfants ayant un historique de TDL (Clegg, Hollis, Mawhood, \& Rutter, 2005; Conti-Ramsden \& Botting, 2008; Johnson, Beitchman, \& Brownlie, 2010; Toseeb, Pickles, Durkin, Botting, \& Conti-Ramsden, 2017). Toutefois, plusieurs facteurs qui réduisent ces impacts néfastes ont été identifiés dans la trajectoire des enfants TDL, tels que l'identification précoce du TDL et une prise en charge appropriée, un bon soutien parental, la disponibilité d'aménagements pédagogiques adaptés, un haut sentiment d'autoefficacité, un bon niveau de prosocialité et de bonnes capacités de lecture (Botting, Bean-Ellawadi, \& Williams, 2016; Conti-Ramsden, Durkin, Toseeb, Botting, \& Pickles, 2018; Parsons, Schoon, Rush, \& Law, 2011; Toseeb et al., 2017). Selon Durkin et Conti-Ramsden (2010), la disponibilité du support offert aux enfants ayant un TDL influence probablement leur devenir, tout comme la façon dont ces jeunes se perçoivent, arrivent ou non à compenser leurs difficultés langagières et vivent des expériences positives ou négatives. En résumé, les besoins de ces enfants justifient des interventions qui s'intéressent à leurs habiletés langagières, mais également à leur développement global et à leur épanouissement personnel.

Malgré les impacts fonctionnels importants auxquels elles font face, les personnes aux prises avec un TDL peinent à accéder aux services dont elles ont besoin. Au Québec, le nombre d'enfants en attente d'évaluation ou de réadaptation est élevé et les délais sont souvent très longs. Selon un rapport de l'Institut national d'excellence en santé et en services sociaux 
(INESSS), 1675 enfants présentant une déficience du langage, dont un TDL, attendaient pour recevoir un premier service spécialisé de réadaptation au 31 mars 2015. Selon les données recueillies, le délai moyen d'attente serait estimé de quatre à six mois, avec des disparités interrégionales importantes (Tessier \& Valade, 2017).

Ce ne sont pourtant pas tous les enfants présentant une problématique langagière qui sont orientés vers ces services. Ainsi, une méta-analyse a révélé que $81 \%$ des enfants de cinq à douze ans consultant pour des problèmes affectifs et comportementaux ont un déficit langagier significatif non identifié (Hollo, Wehby, \& Oliver, 2014), entraînant des conséquences importantes pour eux-mêmes et leurs familles et remettant en question la pertinence des interventions qui leur sont offertes. De façon générale, le TDL est un trouble neurodéveloppemental peu connu du public et même des dispensateurs de services. Les publications scientifiques s'intéressant au TDL sont moins nombreuses que celles s'intéressant à des troubles dont la prévalence ou les impacts sont comparables. Ceci n'est pas étonnant considérant que la recherche sur le TDL serait peu financée (p. ex., 19 fois moins que le trouble du déficit de l'attention avec ou sans hyperactivité [TDAH] en 2008-2009; Bishop, Clark, Conti-Ramsden, Norbury, \& Snowling, 2012).

Le manque de constance des définitions employées pour décrire les troubles du langage et les mésententes entourant les critères d'identification pourraient être partiellement responsables de la méconnaissance de cette problématique (Bishop, 2017). Le langage se trouvant au croisement de plusieurs disciplines, la conceptualisation de ces troubles varie selon les professions et peut être hermétique selon les contextes. Par exemple, les systèmes internationaux de classification biomédicale, tels que le Manuel diagnostique et statistique des troubles mentaux (DSM-5; American Psychiatric Association [APA], 2015) ou la Classification internationale $d u$ fonctionnement, du handicap et de la santé; version pour enfants et adolescents (CIF-EA; Organisation mondiale de la santé [OMS], 2007), seraient utilisés par les professionnels de la santé, mais rejoindraient peu les intervenants du milieu de l'éducation qui cherchent pourtant à soutenir au quotidien les jeunes aux prises avec un TDL (Bishop et al., 2016). Même chez les spécialistes du langage, la terminologie et les définitions employées en clinique et en recherche ont grandement varié au fil des années, tant dans les milieux anglophones (p. ex. specific ou primary language impairment (S/PLI), language impairment, language disorder; Reilly, Bishop, \& Tomblin, 2014) que francophones (p. ex., audimutité, syndrome dysphasique, dysphasie, trouble spécifique du langage oral, trouble primaire du langage; Maillart, 2018;
Smolla et al., 2015). Dans le chapitre Troubles $d u$ langage oral et dysphasies du manuel " Neuropsychologie de l'enfant et de l'adolescentTroubles développementaux et de l'apprentissage $\left(3^{\mathrm{e}}\right.$ édition) », les auteures font d'ailleurs ressortir cette hétérogénéité :

En France [...], le diagnostic de trouble spécifique du langage oral (TSLO) et de dysphasie est utilisé de manière interchangeable alors qu'au Québec, les orthophonistes préfèrent utiliser le terme trouble primaire du langage oral plutôt que le mot dysphasie, terme qui est davantage utilisé par les neuropsychologues. (Lussier, Chevrier, \& Gascon, 2018, p. 257)

En plus de la confusion entraînée par la multiplicité des termes employés, la notion même d'un trouble du langage décrit comme étant "spécifique " a été mise en doute. En effet, il serait impossible d'identifier un TDL (ou dysphasie) par des caractéristiques qui seraient pathognomoniques et qui permettraient de le distinguer d'un autre type d'atteinte langagière. Par exemple, les caractéristiques langagières habituellement attribuées au TSA ne sont pas spécifiques à ce trouble (Gernsbacher, Morson, \& Grace, 2016). Une méta-analyse publiée en 2015 qui s'intéressait à cette question n'a pu soutenir qu'un profil langagier spécifique permettait de caractériser les enfants ayant un TSA, ni les distinguer sur cette base des enfants ayant un TDL (Kwok, Brown, Smyth, \& Cardy, 2015).

Aussi, la notion de spécificité visant à exclure la présence d'autres difficultés (cognitives ou autres) chez l'enfant s'est avérée ne pas être soutenue par la recherche. En effet, le taux élevé de comorbidité ou de chevauchement du trouble du langage avec d'autres troubles neurodéveloppementaux tels que le trouble développemental de la coordination (association de 40-90 \%; Hill, 2001), le TDAH (près de la moitié selon Gillberg et al., 2004), le trouble du spectre autistique (Leyfer, Tager-Flusberg, Dowd, Tomblin, \& Folstein, 2008) ainsi que les troubles spécifiques d'apprentissage (Bishop \& Rutter, 2008; Gooch, Hulme, Nash, \& Snowling, 2014; Snowling, Duff, Nash, \& Hulme, 2016) est bien documenté. Enfin, les diverses nomenclatures proposées (p. ex., Gérard, 1993; Rapin \& Allen, 1983) pour caractériser les profils d'atteintes et les regrouper en sous-types se sont révélées ne pas être stables dans le temps (ContiRamsden \& Botting, 1999). Ces sous-types (p. ex., déficits lexical-syntaxique, phonologique-syntaxique, sémantique-pragmatique) refléteraient donc des variabilités développementales plutôt que de réels profils distincts. Depuis plus de 25 ans, ces différents constats ont amené plusieurs chercheurs à dénoncer la 
notion d'un trouble langagier « spécifique » (Aram, Morris, \& Hall, 1992, 1993; Reilly et al., 2014).

Le rejet de la notion de spécificité de l'atteinte langagière dans le TDL et ce manque d'accord dans la terminologie employée ont mené à la publication en 2014 d'un numéro spécial du International Journal of Language and Communication Disorders (Bishop, 2014; Reilly, Bishop, \& Tomblin, 2014; Reilly et al., 2014). Pour donner suite à cette publication, un groupe de chercheurs britanniques, Bishop et Snowling en tête, a entrepris en 2015 de remédier à cette situation en mettant sur pied le projet CATALISE. Ce projet consistait en une consultation organisée d'experts visant à obtenir un consensus pour une terminologie adéquate. La méthode Delphi a été employée. Répandue dans différents domaines, celleci utilise des questionnaires remplis de façon successive pour colliger des données et fournir une rétroaction auprès d'un groupe d'experts, jusqu'à ce que les convergences soient mises en évidence (pour plus de détails, voir Hsu \& Sandford, 2007). Le panel d'experts constitué se voulait international (six pays anglophones) et multidisciplinaire (57 personnes incluant des orthophonistes, des psychologues, des pédiatres, des psychiatres, des enseignants spécialisés, une audiologiste et des représentants de familles). La première phase des travaux a recherché un consensus sur les critères d'identification (Bishop et al., 2016) et la deuxième a considéré la terminologie, proposant des définitions et une nomenclature standardisée (Bishop et al., 2017).

Bien qu'elle ait été menée en anglais, cette démarche constitue un modèle de référence unique. À la suite de la publication du projet CATALISE, des chercheurs et cliniciens œuvrant en français ont analysé les recommandations proposées puis en ont fait ressortir celles applicables à une langue étrangère, que ce soit en Belgique (Maillart, 2018) ou au Québec (Gingras, 2017). L'OOAQ, après avoir consulté plusieurs membres experts, a adhéré à ce consensus international en septembre 2017, demandant dès lors à ses membres d'adopter la nouvelle appellation et les critères associés au TDL. En octobre 2018, elle a rendu disponible des documents d'information s'adressant au public et aux professionnels de la santé et de l'éducation (www.ooaq.qc.ca), ce qui est dans l'esprit du premier énoncé du consensus. Cet énoncé soutient que l'adoption et l'utilisation d'une terminologie commune par l'ensemble des acteurs concernés par le TDL sont impératives tant pour la recherche que pour la clinique. Il s'agit d'une condition essentielle pour permettre une meilleure intégration des connaissances scientifiques et une meilleure reconnaissance de la problématique, mais également pour favoriser le développement et l'accès aux services nécessaires aux personnes aux prises avec un TDL, sans que cet accès ne soit entravé par des restrictions ou des exclusions administratives non fondées sur les données probantes.

\section{Projet CATALISE : Survol et analyse critique}

Les professionnels souhaitant approfondir leur réflexion peuvent consulter les articles originaux publiés en accès libre par le consortium CATALISE pour prendre acte de la méthodologie employée par les auteurs ainsi que l'ensemble des résultats obtenus. Les 12 énoncés consensuels (plus de $78 \%$ d'accord entre les experts) ont également été traduits et commentés par Mme Marie-Pier Gingras, orthophoniste québécoise, sur le blogue de vulgarisation scientifique Tout cuit dans le bec (Gingras, 2017). Sans prétendre aborder l'ensemble des implications découlant des énoncés de CATALISE, nous choisissons ici de réunir quelques éléments pouvant intéresser plus particulièrement les neuropsychologues. Afin d'ouvrir une discussion interdisciplinaire, l'arbre décisionnel menant à l'identification des troubles du langage, la terminologie proposée, la place des tests standardisés, l'importance des impacts fonctionnels, l'absence de critères d'exclusion, la pertinence d'une évaluation intellectuelle, les principales atteintes langagières à documenter et l'approche basée sur les besoins seront développés.

\section{Arbre décisionnel pour l'identification des troubles du langage}

Tel que mentionné précédemment, la classification des troubles du langage en catégories distinctes a été un écueil au fil du temps puisque celles-ci ne pouvaient être validées et reflétaient plutôt l'évolution du trouble selon l'âge (Conti-Ramsden \& Botting, 1999). De plus, la mésentente concernant les terminologies et ses définitions posait le risque d'entraîner de graves préjudices en excluant certains enfants de l'obtention de services par l'application de critères stricts et non validés. L'arbre décisionnel proposé par le consensus CATALISE, illustré par la Figure 1, suggère plutôt d'identifier les enfants ayant des besoins significatifs et persistants au point de vue du langage, tout en documentant l'hétérogénéité des atteintes. Cette hétérogénéité est reconnue comme faisant partie intégrante du TDL.

\section{Terminologie commune}

Trouble développemental du langage. Le TDL est le terme choisi. Avant de proposer une terminologie unifiée, les experts ont réfléchi aux mots à employer, car ils ont un impact sur la perception du problème langagier. Lorsqu'il y a "trouble » du langage (language disorder), il y a nécessairement à la fois une persistance des difficultés (poor prognosis) et des impacts fonctionnels (everyday functioning) 


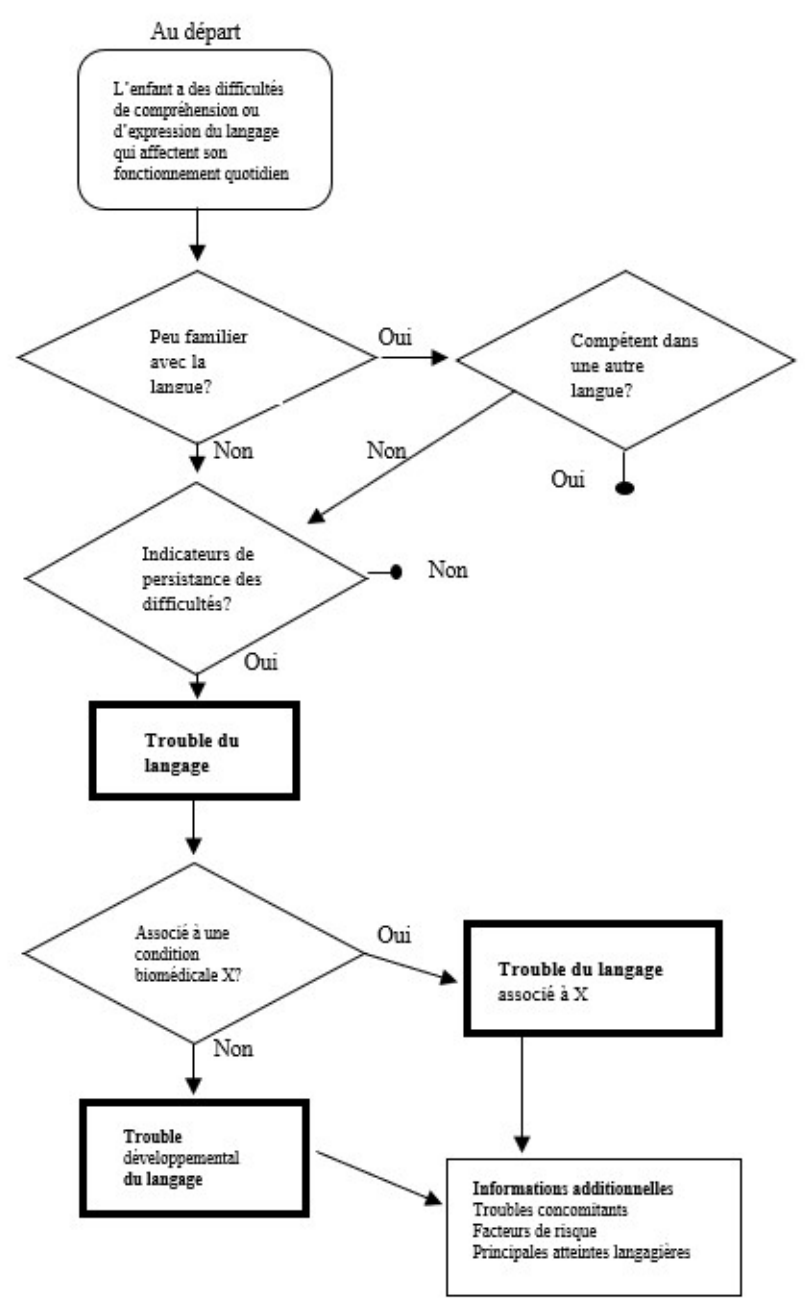

Figure 1. Organigramme illustrant les voies menant au diagnostic du trouble du langage. [Traduction libre] Tiré de l'article de Bishop et al. (2017)

observables. Pour parler d'un trouble dont l'étiologie est incertaine, les experts ont privilégié le terme " développemental " (developmental language disorder; $D L D$ ) à celui de "spécifique » (specific language impairment; $S L I$ ) ou " primaire ». Ceci favorise, entre autres, une cohérence avec la terminologie employée dans d'autres conditions neurodéveloppementales (p. ex., trouble développemental de la coordination, dyslexie développementale) et il s'agit de l'appellation qui a été endossée par la majorité des experts consultés.

Trouble du langage associé à $\mathbf{X}$. Dans le cas où le trouble du langage est associé à une condition biomédicale connue (p. ex., syndrome génétique, TSA, déficience intellectuelle), le terme « développemental» sera substitué par « trouble du langage associé à X». Les experts jugent qu'il est important que le diagnostic de TDL ne concerne que les cas où l'étiologie est incertaine, puisque cela sera utile lors de recherches futures sur ses causes, son pronostic et les interventions à adopter (énoncé 7). Néanmoins, quelques chercheurs américains trouvent que les critères du TDL sont encore trop larges pour permettre des avancées significatives en recherche et critiquent l'abandon du terme SLI, dont les critères d'inclusion et d'exclusion étaient plus stricts (voir ASHA, 2018).

Difficultés de langage. Bien que les capacités langagières de l'enfant soient faibles, le terme " trouble » n'est pas appliqué lorsque des éléments liés à l'enfant ou à son contexte ne permettent pas de penser que les atteintes vont persister. Ainsi, le terme " difficultés de langage " (language difficulties) est recommandé lorsqu'un enfant n'aurait pas été suffisamment exposé à la langue de scolarisation, mais possèderait les habiletés attendues dans une autre langue (énoncé 4). Il est à noter que le terme " difficultés de langage » remplace désormais « retard de langage "(language delay). Ce dernier a été largement rejeté par les experts en raison de la confusion qu'il entraîne et de l'impossibilité de déterminer à l'avance s'il y aura rattrapage, ce qui rend par le fait même illogique l'utilisation du terme " retard ». Dans le cas où un écart du niveau de développement langagier par rapport à la norme est observé chez un enfant très jeune, l'évaluation pourrait mener à conclure à la présence de difficultés langagières, puisque les indicateurs de la persistance des déficits ne sont pas clairs avant quatre ans, selon les connaissances actuelles (voir Tableau 1). Selon Bishop et ses collègues (2017), les données disponibles suggèrent que les indicateurs du pronostic se précisent avec l'âge, mais qu'en général les problèmes affectant un plus grand nombre d'habiletés langagières risquent de perdurer (énoncé 3).

\section{Résultats aux tests standardisés}

L'arbre décisionnel proposé par CATALISE soustend que l'obtention d'un score déficitaire à un test de langage n'est pas suffisant pour conclure à un TDL (Bishop et al., 2017). D'ailleurs, l'écart à la moyenne nécessaire pour établir un score langagier déficitaire n'est pas clairement établi dans la littérature, même si les chercheurs et les cliniciens considèrent généralement qu'une performance inférieure au $10^{\mathrm{e}} \mathrm{ou}$ au $16^{\mathrm{e}}$ rang centile démontre une atteinte langagière significative (Tomblin et al., 1997). En contexte québécois francophone, Thordardottir et al. (2011) ont vérifié quelle était la meilleure combinaison de sensibilité et de spécificité pour identifier une atteinte langagière chez l'enfant de cinq ans, à partir de résultats obtenus à différentes mesures de connaissances et de traitement langagier. Parmi les 92 participants, 14 avaient préalablement été identifiés par une orthophoniste comme ayant un trouble primaire du langage (ancienne appellation du TDL), 
Tableau 1

Indicateurs de persistance des difficultés langagières selon l'âge

3 ans et moins

Données de la littérature

(Selon Bishop et al., 2017)

Indicateurs de persistance

(pronostic moins favorable)

Pas de combinaison de mots

Difficultés de compréhension à 24 mois

Données mitigées

Prédictibilité aug
l'âge

Plus grand nombre de composantes langagières atteintes
À partir de 5 ans

Probabilités faibles que

l'écart avec les pairs ne diminue avec l'âge (Norbury et al., 2016)

Faibles capacités non verbales

Difficultés de compréhension

Difficultés de lecture et d'apprentissage
Indicateurs d'amélioration

(bon pronostic)
Seule la phonologie

expressive est touchée ce qui servait de critère externe. Les épreuves administrées incluaient des tests et sous-tests évaluant le vocabulaire, la morphosyntaxe réceptive, la compréhension de consignes, la répétition de phrases et de non-mots, la dénomination automatique rapide et la répétition de chiffres à l'endroit et à l'envers (voir Thordardottir, Kehayia, Lessard, Sutton, \& Trudeau, 2010; Thordardottir et al., 2011). La sensibilité et la spécificité de ces mesures ont été examinées à trois seuils distincts, préalablement choisis par les auteurs. Ceux-ci ont vérifié le seuil de -1 écart-type $\left(16^{\mathrm{e}}\right.$ rang centile d'une distribution normale), alors fréquemment utilisé en recherche, mais considéré trop laxiste en clinique; le seuil de $-1,28$ écarts-types $\left(10^{\mathrm{e}}\right.$ rang centile), rapporté comme étant fréquemment utilisé en clinique; et le seuil de -2 écarts-types, correspondant au critère officiellement recommandé au Québec à la même époque. Pour distinguer les enfants ayant un TDL de ceux n'en ayant pas, les résultats obtenus ont démontré que le meilleur seuil était au $16^{\mathrm{e}}$ rang centile (-1 écart-type) pour la majorité des mesures employées. Cette étude rappelle que la présence d'un TDL ne correspond pas nécessairement à l'obtention de résultats " sévèrement déficitaires " aux tests standardisés. L'établissement d'un niveau de sévérité des atteintes langagières n'est plus requis pour qualifier un TDL dans une pratique basée sur les données probantes (OOAQ, 2018a). Pourtant, il s'agit d'un critère administratif toujours employé au Québec pour déterminer l'obtention de financement, souvent lié à l'accès aux services. Dans son récent avis concernant l'Organisation du continuum et de la dispensation des services aux enfants âgés de 2 à 9 ans présentant un TDL, l'INESSS a suggéré de réviser ces critères en émettant sa Recommandation 5 :
Le ministère de la Santé et des Services sociaux devrait, de concert avec le ministère de l'Éducation et de l'Enseignement supérieur et le ministère de la Famille, entamer des travaux relatifs à la révision des modes d'allocation des ressources en fonction des besoins de l'enfant et à l'identification des meilleurs prestataires de services, selon les différentes étapes de vie de l'enfant. (Tessier \& Valade, 2017, p. 37)

\section{Impacts fonctionnels}

En continuité directe avec les recommandations du consensus CATALISE, l'OOAQ incite maintenant ses membres à évaluer la sévérité des impacts fonctionnels du TDL dans la vie de tous les jours. Il s'agit d'une approche davantage axée sur les besoins plutôt que sur le diagnostic. En effet, chez deux personnes ayant des incapacités langagières semblables, les répercussions au quotidien peuvent être fort différentes. Afin d'éviter la surmédicalisation, il est recommandé de réserver le diagnostic de TDL aux individus dont les incapacités entraînent des difficultés persistantes dans des domaines variés de leur vie.

Il est donc essentiel de s'intéresser à la façon dont les enfants qui présentent un TDL arrivent ou non à s'adapter aux demandes de leur environnement, à participer pleinement aux activités de la vie quotidienne et à remplir les rôles sociaux attendus pour leur âge. Ceci s'inscrit dans un courant actuel où l'évaluation $\mathrm{du}$ fonctionnement est désormais recommandée comme partie intégrante de toute évaluation faite dans les règles de l'art (Heffer, Barry, \& Garland, 2009). Ce type d'évaluation est même 
intégré dans la démarche pour plusieurs diagnostics de troubles neurodéveloppementaux selon le système de classification du DSM-5 (APA, 2015), tels que le TSA, le TDAH et le trouble spécifique des apprentissages, qui requièrent tous une spécification concernant leur niveau de sévérité en fonction de l'incidence des difficultés et du niveau de soutien nécessaire pour le fonctionnement. Dans le cas du handicap intellectuel, le niveau de sévérité, auparavant déterminé en fonction du niveau intellectuel, est désormais établi à partir du fonctionnement adaptatif. Néanmoins, même si les professionnels de la santé sont de plus en plus sensibilisés à l'importance de considérer la participation sociale de l'individu (Sylvestre, Brisson, Lepage, Nadeau, \& Deaudelin, 2016; Washington, 2009), l'évaluation de l'enfant présentant un TDL s'attarde encore souvent à mesurer les incapacités langagières plutôt que leur impact sur les habitudes de vie (Gomersall et al., 2015; McLeod \& Threats, 2009). Dans le cadre du travail fait par CATALISE, les auteurs soulignent que le manque d'outils valides s'intéressant à l'aspect fonctionnel du langage constitue une des limites importantes aux recommandations. Ils ne proposent toutefois pas de moyens concrets pour y remédier (Bishop et al., 2017).

Aux États-Unis, l'association des orthophonistes préconise l'utilisation du cadre conceptuel du fonctionnement humain proposé par l'OMS dans la CIF-EA (ASHA, 2016). Selon celle-ci, " l'état de fonctionnement et de handicap d'une personne est le résultat de l'interaction dynamique entre son problème de santé (maladies, troubles, lésions, traumatismes, etc.) et les facteurs contextuels "(OMS, 2007). L'OMS préconise donc d'évaluer ces différentes composantes chez l'enfant ou l'adolescent : 1) l'intégrité ou la déficience de ses fonctions organiques et de ses structures anatomiques (p. ex., aires cérébrales activées lors d'une imagerie par résonnance magnétique fonctionnelle [IRMf]); 2) sa capacité ou son incapacité à réaliser des tâches lors d'activités (p. ex., score à une épreuve standardisée du langage); et/ou 3) sa performance à réaliser des tâches dans l'environnement réel permettant ou restreignant sa participation (p. ex., phrases formulées lors de la causerie en classe). Les facteurs contextuels peuvent aussi être recensés : les facteurs environnementaux sont les facilitateurs ou les obstacles externes qui affecteront le fonctionnement et le handicap de l'enfant (p. ex., milieu bruyant, disponibilité d'un support imagé sur le pupitre, accès à une subvention gouvernementale) alors que les facteurs personnels sont les attributs de la personne ayant un impact sur le fonctionnement et le handicap (p. ex., sexe, âge, autres problèmes de santé, niveau d'éducation, tempérament, etc.).
Ainsi, l'évaluation de la participation et des facteurs contextuels peut s'avérer pertinente pour documenter les impacts fonctionnels du TDL. Pour l'instant, selon une récente recension des écrits dont les critères s'appuyaient sur la CIF-EA (OMS, 2007), la majorité $(65 \%)$ des outils d'évaluation employés pour évaluer les progrès d'enfants d'âge préscolaire aux prises avec des problèmes de communication mesurerait des résultats dans les activités (capacités ou incapacités en contexte standardisé), alors que seulement $15 \%$ mesureraient des changements dans la participation (performance dans l'environnement réel) (Cunningham et al., 2017).

Précisons qu'un cadre conceptuel légèrement différent de celui de la CIF-EA est très utilisé en contexte québécois, notamment dans les centres de réadaptation physique où doivent être orientés les enfants présentant une déficience langagière, incluant le TDL (Tessier \& Valade, 2017). Il s'agit du Modèle de développement humain - Processus de production du handicap (MDH-PPH), développé en 1998 et bonifié en 2010 (Fougeyrollas, 2010; Fougeyrollas, Cloutier, Bergeron, Côté, \& St-Michel, 1998). Tout comme la CIF-EA, le MDH-PPH s'appuie sur un modèle systémique multidimensionnel et vise à décrire ainsi qu'à expliquer le phénomène du handicap en tenant compte des facteurs personnels et environnementaux. Selon ses auteurs, le MDH-PPH présente toutefois plusieurs caractéristiques qui le distinguent de la CIF-EA (Fougeyrollas, 2005) et une classification internationale MDH-PPH est disponible depuis 2018 (pour plus de détails, consulter le site du Réseau international sur le Processus de production du handicap : https://ripph.qc.ca/).

Les orthophonistes sont généralement familiers avec le modèle conceptuel du MDH-PPH, mais utilisent peu d'outils standardisés pour documenter les impacts fonctionnels du TDL. Ils recueillent plutôt des informations auprès des proches dans les différents milieux de vie de l'enfant, que ce soit par entrevue, par questionnaires ou par observations directes, pour ensuite intégrer ces éléments à leur impression clinique globale.

Il est tout de même encourageant que certains chercheurs se soient récemment appliqués à développer des moyens d'évaluation ciblant les impacts fonctionnels du TDL (Croteau et al., 2015; Thomas-Stonell, Washington, Oddson, Robertson, \& Rosenbaum, 2013). Il s'agit principalement de questionnaires qui, malgré leur pertinence, sont relativement peu connus ou peu utilisés sur le terrain, soit parce qu'ils sont parfois jugés laborieux ou compliqués à remplir, soit parce qu'ils ne font tout simplement pas partie des habitudes des cliniciens. 
Dans ce contexte, le travail interdisciplinaire peut certainement bonifier le service rendu à l'enfant. Par exemple, l'enseignant et les intervenants scolaires peuvent documenter le fonctionnement de l'enfant en contexte d'apprentissage et de socialisation avec les pairs. Les psychologues peuvent contribuer à documenter l'impact du TDL, notamment sur la régulation émotionnelle, l'estime de soi, la construction de l'identité et la relation parent-enfant. La mesure des comportements adaptatifs, déjà connue des psychologues et neuropsychologues, apparaît également comme une avenue pertinente pour évaluer les impacts fonctionnels du TDL chez l'enfant.

\section{Remplacement des critères d'exclusion}

Reilly et ses collègues (2014) décrivent bien l'histoire et l'évolution des termes employés pour décrire les TDL. Ils soulignent que les critères d'exclusion utilisés ont toujours été sous-tendus par l'idée que les difficultés langagières survenaient en l'absence d'autres déficits. Cependant, les critères d'exclusion ont varié et n'ont pas fait consensus en recherche. Généralement, pour avoir un SLI les participants devaient présenter un niveau de capacités langagières correspondant à un déficit, alors que le potentiel intellectuel non verbal était préservé (QI $\geq$ 85 , écart verbal - non verbal démontré). La présence d'une atteinte non verbale ou de tout autre trouble neurodéveloppemental constituait généralement un critère d'exclusion en recherche, étant plus sévère qu'en clinique. Dans le DSM-IV-TR, le seul critère d'exclusion était la présence d'un trouble envahissant du développement. Dans le DSM-5, les critères d'exclusion ont été abolis. Il y est par ailleurs mentionné « qu'en présence d'un retard mental, d'un déficit moteur affectant la parole, d'un déficit sensoriel ou d'une carence de l'environnement, les difficultés de langage doivent dépasser celles habituellement associées à ces conditions " (APA, 2015, p. 75). Dans le milieu clinique, les critères d'exclusion diagnostiques ont aussi été appliqués de façon variable selon les régions et les époques, en plus d'être fréquemment confondus avec des critères d'exclusion administratifs. Par exemple, au Québec, le Ministère de l'Éducation exige que six mois de suivi orthophonique précède l'octroi d'un code de difficulté langagière (Gouvernement du Québec, 2007). Certains cliniciens en sont venus à exiger l'atteinte de ce critère avant de poser un diagnostic de TDL, alors qu'il ne s'agit pas d'une exigence professionnelle. À l'inverse, certains organismes ont parfois utilisé des critères développés pour la recherche comme exigences au financement (p. ex., commission scolaire refusant de considérer un diagnostic de TDL sans la démonstration d'un écart verbal - non verbal). Dans cette optique, le consensus CATALISE propose de ne plus utiliser de critères d'exclusion pour définir les troubles du langage (énoncé 4), leur usage ayant malheureusement mené à plusieurs dérives, car ils étaient parfois interprétés comme des critères de nonadmissibilité aux services (Bishop et al., 2017). L'identification de conditions biomédicales associées, de troubles concomitants et de facteurs de risque sont cependant proposés pour ne pas amalgamer tous les enfants ayant un problème de langage, sans égard à la cause ou au type d'intervention requis (Bishop et al., 2017).

Conditions biomédicales. La déficience intellectuelle et le TSA ont été inclus dans les conditions biomédicales parce qu'ils seraient communément liés à des causes génétiques ou neurologiques, celles-ci étant de mieux en mieux identifiées grâce aux progrès technologiques. Par exemple, plusieurs études indiquent une très forte composante génétique pour le TSA (Constantino et al., 2013; Mash \& Barkley, 2014). Parmi les autres conditions biomédicales identifiées par le consensus CATALISE, citons les traumatismes crânienscérébraux, les aphasies épileptiques acquises dans l'enfance, la déficience motrice cérébrale, les pertes auditives neurosensorielles ou des conditions génétiques comme le syndrome de Down (Bishop et al., 2017).

Troubles concomitants. $\mathrm{Au}$ contraire des conditions biomédicales, les troubles concomitants sont des déficits cognitifs, sensori-moteurs ou comportementaux, dont l'étiologie apparaît moins claire, complexe ou multifactorielle selon les connaissances actuelles. Les troubles concomitants incluent les diagnostics et les difficultés qui peuvent affecter le type de déficits langagiers et la réponse de l'enfant à l'intervention, sans qu'une relation causale évidente ne puisse être établie avec le trouble du langage (énoncé 9). Bishop et ses collègues (2017) incluent dans cette catégorie le TDAH, le trouble développemental de la coordination (TDC), la dyslexie-dysorthographie ainsi que les problèmes émotionnels et comportementaux. Les troubles de la parole qui touchent le pôle moteur (par exemple la dyspraxie verbale, les troubles de l'articulation, la dysarthrie et le bégaiement) peuvent aussi être des troubles concomitants au TDL. Les troubles de traitement auditif auraient pu être considérés comme tels, mais n'ont pas été inclus dans l'énoncé consensuel en raison de la controverse entourant ce diagnostic en soi (Bishop et al., 2017).

Plusieurs de ces troubles comportent des caractéristiques pouvant être intrinsèquement liées au TDL. Par exemple, des déficits de la mémoire de travail se retrouvent fréquemment chez l'enfant présentant un TDL, qu'il y ait ou non un TDAH concomitant. Le neuropsychologue observant des 
difficultés liées à la mémoire ou au traitement verbal se gardera de conclure à la présence de l'un ou de l'autre diagnostic avant d'avoir affiné son analyse ( $p$. ex., en comparant avec le traitement visuel, en vérifiant les autres capacités attentionnelles et langagières, en se concertant avec l'orthophoniste). Il en est de même pour les difficultés de lecture et d'écriture pouvant être observées chez l'enfant ayant un TDL, que celui-ci présente ou non un trouble spécifique des apprentissages comorbide. Les capacités langagières étant nécessaires tant dans la modalité orale qu'écrite, des déficits de compréhension ou d'expression touchant le traitement de l'information verbale, la phonologie, la syntaxe, le vocabulaire, le discours ou la pragmatique peuvent se répercuter sur la lecture et l'écriture. Selon le profil, les atteintes peuvent être variables selon les individus et différer à l'oral et à l'écrit. Par exemple, un enfant ayant un TDL pourrait obtenir de bons résultats aux tests de vocabulaire et de syntaxe à l'oral, mais se trouver en difficulté significative dans ces domaines à l'écrit, où le registre attendu est généralement plus abstrait, élaboré et complexe. En dictée, un enfant confronté à des mots complexes mal maîtrisés à l'oral risque fort de commettre des erreurs d'orthographe lexicale. Ainsi, une analyse approfondie sera nécessaire pour déterminer si les déficits de vitesse, de précision ou de compréhension de lecture, d'orthographe ou de rédaction que présente un élève sont mieux expliqués par un TDL (connu ou non diagnostiqué), un trouble spécifique des apprentissages, la concomitance de ces deux troubles ou par d'autres facteurs. Au-delà des étiquettes, une bonne collaboration interprofessionnelle permettra de mieux identifier les zones de forces et de difficultés spécifiques à l'élève et de proposer les pistes de solution les plus efficaces pour lui. Il arrive fréquemment que le neuropsychologue soit le premier interpellé pour évaluer un enfant confronté à des difficultés d'apprentissage. S'il est sensibilisé aux diverses manifestations possibles du TDL à l'oral et à l'écrit, son interprétation du profil sera déterminante et il référera en orthophonie, alors que la famille ou l'école n'avait pas envisagé cette avenue.

Le fait que la présence de troubles concomitants n'exclue pas le diagnostic de TDL représente certainement mieux la réalité clinique que les " cas purs " décrits antérieurement par l'étiquette de SLI, dont les nombreux facteurs d'exclusion rendaient les résultats difficilement généralisables aux populations consultantes. Si les professionnels observent que les enfants TDL ont la plupart du temps diverses difficultés (Benner, 2005; Cohen, Davine, \& MelocheKelly, 1989; Hill, 2001), Bishop et ses collègues (2017) soulignent qu'un même enfant peut actuellement porter plusieurs étiquettes diagnostiques selon la perspective adoptée par les différents professionnels, créant faussement l'impression qu'il présente plusieurs conditions distinctes. À cet effet, l'article de Bishop et al. (2017) sur le projet CATALISE présente un exemple de cas semblable à ceux rencontrés à maintes reprises lors de nos propres expériences cliniques en pédopsychiatrie : un enfant présentant un TDL selon l'orthophoniste a également une dyslexie selon un neuropsychologue, un trouble $\mathrm{du}$ traitement auditif selon l'audiologiste, un TDAH identifié par un pédiatre et/ou un trouble de l'opposition avec provocation diagnostiqué par un psychiatre. Étant donné la subjectivité et les limites de ces différentes catégories diagnostiques, il apparaît effectivement plus prudent de ne pas exclure un diagnostic de TDL en raison de la présence d'une autre étiquette de trouble neurodéveloppemental dont l'étiologie, commune ou non, n'a pas encore été établie. Ceci d'autant plus dans le contexte où la cooccurrence entre les troubles neurodéveloppementaux est de plus en plus considérée comme étant une caractéristique intrinsèque de cette famille de troubles (APA, 2015; Bishop \& Rutter, 2008; Gillberg, 2010).

Facteurs de risques. Certains facteurs environnementaux ou biologiques (p. ex., être un garçon, avoir une histoire familiale de troubles du langage ou des apprentissages, avoir vécu des difficultés périnatales, être le plus jeune enfant d'une famille nombreuse ou avoir une mère peu scolarisée) sont considérés des facteurs de risque parce qu'ils sont plus fréquemment observés chez les enfants TDL que chez les enfants dont le développement est typique. Bien qu'ils soient statistiquement associés aux troubles du langage, ces facteurs n'ont pas de relations causales bien établies selon la littérature actuelle. La présence de ces facteurs de risque n'exclut pas la présence d'un diagnostic de TDL chez l'enfant (énoncé 10).

\section{Évaluation intellectuelle}

Pas d'écart verbal - non verbal nécessaire. Pour rencontrer les critères d'exclusion décrits plus haut, il était habituellement convenu d'examiner le fonctionnement intellectuel de l'enfant. Si un profil de compétence indiquait la présence de difficultés plus spécifiques à la sphère verbale en présence de compétences non verbales mieux préservées, il permettait d'appuyer la notion d'une atteinte spécifique de la fonction langagière dans le développement de l'individu. Cette tradition a pu ainsi alimenter une approche interprétative du fonctionnement intellectuel fondée sur l'analyse des forces et des faiblesses d'un individu (Jacobs, 2017). La nécessité diagnostique d'observer un écart entre les compétences langagières déficitaires et le fonctionnement non verbal de la personne (p. ex., 
raisonnement logique à partir d'images) fait objet de débats animés depuis plusieurs années, tant en ce qui concerne les troubles spécifiques d'apprentissage que le TDL (Aram et al., 1992; Braden \& Weiss, 1988). De plus, des études longitudinales ont observé de très grandes fluctuations du QI non verbal selon le niveau développemental de l'enfant, dont une chute massive d'environ 20 points entre 8 et 11 ans (Conti-Ramsden, St Clair, Pickles, \& Durkin, 2012). Hormis ces données empiriques, la pratique de l'utilisation de l'écart verbal - non verbal persiste dans la pratique clinique et devrait être questionnée (Lyons et al., 2008). Plusieurs questions demeurent également en attente de réponses dans l'interprétation de cet écart. Par exemple, dans le cas où les capacités non verbales se situent au niveau de la douance et les capacités verbales dans la moyenne, est-il indiqué de considérer le verbal comme une faiblesse relative nécessitant du support, ou plutôt le non verbal comme une force? Ces aspects ne sont pas abordés dans le projet CATALISE, et mériteraient également d'être débattus dans un contexte interdisciplinaire. Toutefois, il est clair que le DSM-5 exige un déficit des capacités langagières par rapport au niveau attendu pour l'âge et non relativement à l'ensemble du profil pour le diagnostic de TDL. Certains auteurs mettent également en garde les cliniciens d'identifier comme problématiques des zones de faiblesses relatives dans un profil globalement élevé, où le fonctionnement est tout de même au niveau ou même au-dessus du niveau de fonctionnement des pairs (Flanagan \& Alfonso, 2017).

Selon la nomenclature du DSM-5, une différence significative entre le potentiel intellectuel non verbal et le rendement académique ou le niveau langagier n'a pas à être observée pour diagnostiquer les troubles spécifiques d'apprentissage ou le TDL. Pour donner suite à ces modifications amenées au DSM-5, Norbury et ses collègues (2016) ont vérifié dans un échantillon populationnel britannique quel impact l'inclusion d'enfants avec des habiletés non verbales variables pouvait avoir sur la prévalence, la présentation clinique et les retombées fonctionnelles du trouble du langage à l'entrée à l'école. Les enfants TDL ayant des habiletés non verbales sous la moyenne (sans atteindre le seuil de la déficience intellectuelle; entre -2 et -1 écarts-types) constituaient $2,78 \%$ de la population alors que les enfants TDL ayant des habiletés non verbales dans la moyenne $(>-1$ écarttype) en représentaient $4,80 \%$. Ces deux sous-groupes ne se distinguaient pas quant à l'ampleur des déficits langagiers, des difficultés scolaires ou des problèmes émotionnels ou comportementaux (Norbury et al., 2016). Bien que les critères administratifs aient fréquemment fait du potentiel non verbal un critère d'exclusion aux services professionnels et éducatifs alloués aux enfants TDL, il n'y aurait aucune raison évidente d'exclure les enfants ayant des habiletés non verbales sous la moyenne selon les auteurs de cette étude. Cette conclusion a été adoptée par les experts consultés dans le cadre du projet CATALISE (Bishop et al., 2017), indiquant que l'enfant avec un TDL peut avoir un faible niveau d'habiletés non verbales (énoncé 8).

Profil cognitif utile à l'intervention. L'évaluation psychologique ou neuropsychologique peut être pertinente à plusieurs enfants présentant des difficultés langagières, pour bien d'autres raisons que la mesure d'un écart entre les habiletés verbales et non verbales. Sachant que le TDL est très fréquemment associé à la présence d'autres problématiques telles que des problèmes de comportements, le TDAH, le TDC et les troubles spécifiques des apprentissages, il est important de considérer le profil cognitif global de l'enfant si des difficultés de cet ordre sont soupçonnées. Le neuropsychologue est souvent le premier interpellé pour documenter le fonctionnement cognitif, ainsi que pour $y$ relier les impacts fonctionnels observés.

De même, les orthophonistes et autres professionnels intervenant auprès d'un enfant qui semble présenter des capacités intellectuelles en deçà des attentes vont continuer de le référer en psychologie ou en neuropsychologie. Dans le cas où le trouble du langage est associé à une déficience intellectuelle ou à une autre condition, l'organigramme diagnostique proposé par Bishop et ses collègues (2017) suggère de le spécifier en considérant qu'il s'agit d'une condition biomédicale pouvant nécessiter une intervention spécifique (énoncé 6). Selon Norbury et ses collègues (2016), la prévalence du trouble du langage associé à une déficience intellectuelle et/ou à une condition médicale préalablement diagnostiquée serait de $2,34 \%$, ce qui augmente la prévalence totale du trouble du langage à $9,92 \%$ des enfants de quatre à cinq ans. Chez les enfants dont le trouble du langage est associé à une déficience intellectuelle ou à une autre condition médicale, les déficits langagiers mesurés seraient globalement plus sévères et une plus grande proportion présenterait un niveau clinique de problèmes sociaux, émotionnels et comportementaux (Norbury et al., 2016). En ce sens, il paraît important de souligner dans l'évaluation de ces enfants qu'ils auront besoin de services en raison d'un trouble du langage associé à X.

\section{Principales atteintes langagières à documenter}

La démarche diagnostique décrite dans le consensus CATALISE nécessite donc d'établir la présence de déficits touchant l'expression et/ou la compréhension du langage, de juger que ces difficultés risquent d'être persistantes et qu'elles ont un impact significatif sur le fonctionnement de 
l'enfant pour conclure à un trouble développemental du langage ou, le cas échéant, à un trouble du langage associé à une condition biomédicale $\mathrm{X}$. Dans le cas d'un TDL, les troubles concomitants et les facteurs de risque identifiés doivent être précisés.

Conscients de l'hétérogénéité des présentations du TDL, les experts du consensus CATALISE proposent aux cliniciens de préciser les domaines langagiers devant faire l'objet d'une intervention, ou, pour les chercheurs, les critères permettant de constituer des échantillons moins hétérogènes dans une étude (énoncé 11). Dans cette optique, Bishop et ses collègues (2017) incluent des lignes directrices pour évaluer le langage de façon plus approfondie. Bien qu'ils mentionnent que la lecture et l'écriture soient fréquemment atteintes dans le TDL, les auteurs ont centré leurs recommandations sur l'évaluation de la modalité orale du langage. Selon eux, la distinction traditionnellement utilisée dans le DSM-IV entre les déficits touchant la sphère expressive ou la sphère réceptive est insuffisamment précise. Cette distinction a d'ailleurs été abandonnée dans le DSM-5 (APA, 2015), qui mentionne également l'hétérogénéité des atteintes. Pour mieux indiquer quels aspects du langage sont problématiques, Bishop et ses collègues (2017) recommandent d'analyser et de caractériser chacun des domaines suivants : la phonologie, la syntaxe, la sémantique/l'accès lexical, la pragmatique/ l'utilisation du langage, le discours ainsi que la mémoire/l'apprentissage verbal(e).

\section{Approche basée sur les besoins}

Tel qu'illustré dans la Figure 2, le TDL peut être conceptualisé comme faisant partie d'un ensemble plus large de difficultés touchant la communication, la parole et le langage. En effet, d'autres enfants que ceux présentant un TDL peuvent avoir besoin de services en orthophonie. Le terme Speech, Language and Communication Needs est déjà employé en éducation en Grande-Bretagne et recommandé par les experts de CATALISE pour offrir la reconnaissance nécessaire aux enfants qui ne présentent pas nécessairement de TDL, mais bénéficient de support spécifique en lien avec la communication, la parole ou le langage (énoncé 12; Bishop et al., 2017). À notre connaissance, il n'existe pas de terme universel équivalent en français, du moins en usage au Québec. Il serait pourtant pertinent d'y voir, à l'heure où les services aux plans de la communication et du langage doivent être repensés au-delà de la réadaptation individuelle et être réfléchis dans un contexte de santé publique (Law, Reilly, \& Snow, 2013). Sans nier le besoin de services spécifiques évident pour plusieurs individus, notamment ceux aux prises avec un TDL, il faut aussi prévoir une terminologie permettant d'identifier et de reconnaitre les besoins populationnels.

Au Québec, les besoins populationnels sont de plus en plus reconnus, notamment par le modèle de réponse à l'intervention. Celui-ci est maintenant répandu en milieu scolaire et démontre bien la nécessité



Figure 2. Diagramme de Venn illustrant la relation entre le TDL et différents termes de diagnostic. [Traduction libre] Tiré de l'article de Bishop et al. (2017). 
d'intervenir sur un continuum pour prévenir l'échec (Barnett, Neely, Wolsing, \& Bunger, 2006; BrownChidsey \& Steege, 2011). Utilisant une pyramide en trois niveaux, le modèle de réponse à l'intervention est conçu pour améliorer la réussite de tous les élèves. Il planifie l'emploi de méthodes validées par la recherche auprès de tous ainsi que des dépistages systématiques (niveau 1), l'offre d'interventions supplémentaires plus spécifiques auprès de petits groupes (niveau 2), dont le suivi des progrès décidera de la poursuite d'interventions intensives adaptées aux besoins de l'élève en modalité individuelle si nécessaire (niveau 3). Cette approche est « destinée à contrer le phénomène de l'intervention tardive auprès des élèves en difficulté » (Lévesque, 2017, para. 1). Le récent avis publié par l'INESSS sur l'Organisation $d u$ continuum et de la dispensation des services aux enfants âgés de 2 à 9 ans présentant un trouble développemental $d u$ langage (trouble primaire $d u$ langage) recommande également de structurer les services selon trois niveaux imbriqués : universel, ciblé et spécialisé, sans toutefois qu'il n'y ait aucun dépistage systématique des problèmes de développement du langage (Tessier \& Valade, 2017). Dans cette perspective, le neuropsychologue cernant des besoins touchant la communication, la parole ou le langage ne devrait pas hésiter à référer l'enfant vers des services appropriés, que celui-ci ait ou non un TDL.

\section{Arrimage de la nouvelle terminologie : défis et ressemblances}

Le consensus international et interdisciplinaire du groupe CATALISE concernant la terminologie et les critères diagnostics du TDL se superpose à d'autres terminologies existantes, dont la plus reconnue en Amérique du Nord est probablement celle du DSM-5 (APA, 2015). Bien que des défis d'arrimage inhérents aux différences de vocabulaire soient présents, un examen plus attentif de leurs contenus permet d'y trouver plusieurs points communs.

Le diagnostic de trouble du langage fait partie des troubles de la communication selon le DSM-5 (APA, 2015), catégorie qui inclut également le trouble de la phonation (articulation des phonèmes), le trouble de la fluidité verbale (bégaiement), le trouble de la communication sociale (pragmatique) et le trouble de la communication non spécifié. La définition du DSM-5 indique que le trouble du langage (dans ses différentes modalités : oral, écrit, signé ou autre) consiste en des difficultés persistantes d'acquisition et d'utilisation du langage (critère A). Cela peut inclure un vocabulaire restreint, une carence de structuration des phrases ou une déficience dans le discours. Les atteintes langagières doivent être marquées et les capacités doivent être quantifiables, inférieures au niveau attendu pour l'âge et résulter en des limitations fonctionnelles dans une ou différentes sphères de fonctionnement (communication, socialisation, scolarisation, rendement professionnel) (critère B). Tel qu'indiqué plus haut, il est reconnu que les capacités expressives et réceptives peuvent être affectées selon une gravité variable. Le DSM-5 note également que les difficultés de compréhension sont souvent sousestimées étant donné la capacité de l'enfant à déduire le sens à partir de ses observations du contexte, alors qu'elles confèrent un moins bon pronostic. Les atteintes de mémoire verbale sont également mentionnées. Les symptômes doivent avoir débuté dans la période précoce du développement (critère C) et ne doivent pas pouvoir être expliqués par un déficit physique (auditif, sensoriel, moteur cérébral ou autre affection neurologique ou médicale) ou intellectuel. Dans ce dernier cas, un diagnostic de trouble du langage est posé lorsque les atteintes langagières sont clairement au-delà des limitations intellectuelles.

Tout comme relevé par les auteurs de CATALISE, le DSM-5 précise que la compétence langagière est plus stable et prédictive à partir de l'âge de quatre ans, mais que le profil de forces et difficultés est susceptible de changer au cours du développement de l'enfant. De plus, tel qu'indiqué plus haut, au-delà des considérations particulières en présence d'un handicap intellectuel ou de retard global de développement, le DSM-5 ne fait aucune mention quant à la recherche d'un écart entre les compétences verbales et non verbales de l'enfant. Seuls des résultats de scores à des tests standardisés de langage, intégrés à une démarche évaluative qui inclut l'anamnèse et des observations cliniques, sont nécessaires pour le diagnostic. Le DSM-5 indique aussi que les scores aux tests standardisés pourraient orienter l'estimation de la sévérité du trouble, sans précision supplémentaire. Enfin, la présence du critère $B$ implique que les limitations dans les sphères de fonctionnement de l'enfant doivent être mesurées. Ceci est cohérent avec l'idée globale véhiculée par le DSM-5 stipulant que tout diagnostic posé doit entraîner des difficultés de fonctionnement de l'individu, sans quoi les symptômes observés ne sont pas considérés suffisamment sévères pour justifier un diagnostic. Toutefois, contrairement à ce qui est prévu pour d'autres troubles neurodéveloppementaux tels que le retard mental, le TSA et les troubles spécifiques des apprentissages, il n'y a aucune définition proposée afin d'en qualifier la sévérité. Ainsi, les critères diagnostiques du DSM-5, le consensus CATALISE et les directives de l'OOAQ convergent concernant la nécessité de documenter le fonctionnement en plus de la stricte évaluation langagière, même si l'objectif dans le cas du DSM-5 est diagnostique, alors qu'il vise à documenter la sévérité du trouble dans le cas de l'OOAQ. 
Ainsi, le trouble du langage tel que défini par le DSM-5 rejoint la définition du TDL en étant distinct d'une affection liée à un problème physique ou neurologique (Bishop et al., 2017). Un autre point commun entre ces deux terminologies est qu'elles ne cherchent ni l'une ni l'autre à caractériser des soustypes. Par ailleurs, tel que mentionné plus haut, le DSM-5 considère comme étant inhérente la comorbidité entre les troubles neurodéveloppementaux. En revanche, il ne mentionne pas les nombreuses difficultés psychosociales associées au TDL ni la comorbidité très élevée avec des troubles comportementaux et affectifs tels que le trouble oppositionnel, le trouble des conduites ainsi que l'anxiété sociale.

Par ailleurs, certaines contradictions peuvent être relevées entre le DSM-5 et l'avis des experts du consensus CATALISE. Par exemple, le diagnostic de trouble de communication sociale (TCS), apparu dans le DSM-5, a été considéré, mais non retenu par les experts du consensus CATALISE. La définition du TCS dans le DSM-5 sous-tend qu'il s'agit d'un trouble différent des troubles du langage, alors que Bishop et ses collaborateurs (2017) considèrent la pragmatique comme une composante faisant partie intégrante du langage. Cette divergence d'opinion est brièvement relevée dans l'article de Bishop et al. (2017), mais il est à souhaiter que le sujet soit développé davantage au cours des prochaines années.

En neuropsychologie, Lussier, Chevrier et Gascon (2018) soulignent dans leur chapitre consacré aux troubles du langage oral qu'aucune classification n'a encore fait consensus, mais prennent soin d'en évoquer plusieurs. En plus du modèle du DSM-5 discuté ci-haut, les auteurs citent dans la troisième édition de leur ouvrage une définition du trouble primaire du langage (dysphasie) proposée par l'OOAQ à ses membres en 2005. Ce n'est qu'en octobre 2018 que l'OOAQ a diffusé une mise à jour par le biais du dépliant Trouble développemental $d u$ langage (TDL) - Fiche explicative pour les professionnels (disponible sur le site de l'Ordre des orthophonistes et audiologistes du Québec : www.ooaq.qc.ca). Lussier, Chevrier et Gascon (2018) ont aussi fait le choix de présenter à leurs lecteurs une " classification des dysphasies ", tout en notant que l'utilisation d'une typologie des dysphasies basée sur les symptômes de l'enfant était remise en question. En milieu clinique québécois, ce type de catégories n'est plus employé par les orthophonistes depuis de nombreuses années, ce qui peut engendrer des défis d'arrimage sur le terrain.

Bien qu'exprimées différemment, plusieurs pratiques cliniques auprès des personnes atteintes d'un TDL semblent déjà être recommandées unanimement, que ce soit en neuropsychologie, en orthophonie ou en psychiatrie (voir Tableau 2). Par ailleurs, quelques pratiques cliniques divergentes ou non consensuelles peuvent être identifiées et constituent des défis d'arrimage pouvant potentiellement nuire à la qualité des services offerts (voir Tableau 3).

\section{Conclusion : importance et retombées cliniques}

En somme, la publication récente d'un consensus interdisciplinaire et international concernant le diagnostic et la terminologie rattachée au TDL constitue une avancée significative dans ce domaine. Motivé par le besoin de mieux sensibiliser la population à ce trouble si prévalent ainsi que par la nécessité de mieux identifier les personnes qui vivent avec un TDL et en subissent les impacts dans leur vie quotidienne, le projet CATALISE s'appuie sur une méthodologie scientifique rigoureuse faisant appel aux données probantes. Il est à souhaiter que l'adoption d'une appellation et de critères communs associés au TDL ait également des retombées positives sur la recherche. Si le TDL et ses impacts sont mieux connus et reconnus, il est possible que des chercheurs de différentes disciplines s'y intéressent et que davantage de financement y soit accordé. Ceci a déjà été observé pour d'autres troubles ayant une prévalence et des impacts semblables dans la population, tel que le TDAH (Bishop, 2010).

L'orthophonie ainsi que la neuropsychologie, deux professions amenées à identifier et soutenir les individus aux prises avec un TDL, se sont développées depuis une soixantaine d'années au Québec, avec une croissance accélérée au cours des deux dernières décennies. Contrairement au domaine des troubles acquis, l'étiologie et l'évolution des TDL sont beaucoup moins connus, et beaucoup de chemin reste à parcourir pour développer et valider les interventions les plus efficaces auprès de cette population. Pour y arriver, la concertation entre les différents acteurs impliqués, y compris les personnes atteintes et leurs proches, est une condition préalable essentielle.

La discussion amorcée entre chercheurs devrait se poursuivre entre cliniciens et entre ordres professionnels, mais également avec les étudiants universitaires des différents domaines, les associations d'usagers, les décideurs, les dispensateurs de services ainsi que le grand public. En ce sens, une mise à jour des critères d'accès aux services pour les enfants TDL, que ce soit dans le milieu de la santé ou de l'éducation, est absolument nécessaire. À titre d'exemple, c'est en 2007 que le Ministère de l'Éducation et de l'Enseignement supérieur du Québec (alors appelé le Ministère de l'Enseignement, des loisirs et du sport; MELS) a actualisé pour la dernière fois son organisation des services éducatifs aux élèves à risque et aux élèves handicapés ou en difficulté 
Tableau 2

Pratiques cliniques auprès des individus ayant un TDL recommandées par plusieurs disciplines

\begin{tabular}{ll}
\hline Pratique clinique & Caractéristiques du TDL faisant consensus \\
\hline Caractérisation des atteintes langa- & -Peuvent toucher différentes modalités (p. ex., orale, écrite, langage des signes) \\
gières & -Peuvent toucher l'expression et la compréhension \\
& -Peuvent toucher différentes composantes : phonologie/production des sons; morpho- \\
& syntaxe; sémantique; vocabulaire; discours
\end{tabular}

Recherche de critères diagnostiques liés à la persistance des atteintes et à l'impact sur le fonctionnement de la personne

Vérification de la présence d'une condition biomédicale, de troubles concomitants ou de facteurs de risque associés

Établissement des recommandations et des interventions nécessaires
-La persistance dans le temps peut être avérée ou jugée probable

-Il est attendu que le portrait clinique varie pour un même individu selon l'âge; les atteintes ne seront pas nécessairement les mêmes au fil du temps -L'impact des atteintes langagières sur le fonctionnement de l'individu peut être observable aux plans des interactions sociales, des apprentissages, des activités professionnelles ou des habitudes de vie

-Une condition biomédicale telle qu'une déficience auditive, une déficience intellectuelle, un syndrome génétique ou une lésion cérébrale doit être identifiée -Plusieurs troubles sont fortement associés au TDL, incluant le TDAH, le TDC, les troubles spécifiques des apprentissages (dyslexie-dysorthographie), les difficultés exécutives et comportementales

-Il faut documenter les antécédents familiaux et les conditions environnementales adverses ou favorables

-L'intervention est essentielle à l'évolution

-Il est nécessaire de considérer le portrait global des forces et des difficultés de la personne

Note. Selon le Manuel Diagnostique et Statistique des Troubles Mentaux (APA, 2015), le manuel Neuropsychologie de l'enfant et de l'adolescent (Lussier, Chevrier et Gascon, 2018) et la Fiche d'avancement de la pratique orthophonique suivant CATALISE (OOAQ, 2018)

Tableau 3

Pratiques cliniques désuètes ou non consensuelles auprès des enfants ayant un TDL

Pratique clinique
-Emploi de termes spécifiques ayant un sens différent selon
les disciplines (p. ex., trouble de la phonation selon le
DSM-5 correspondant au trouble du développement des sons
de la parole selon l'OOAQ ou à la dyspraxie verbale selon
Lussier, Chevrier et Gascon (2018)
-Emploi d'une sémiologie basée sur les symptômes de l'en-
fant (p. ex., déficit syntaxique phonologique; syndrome lexi-
cal syntaxique)
-Emploi de termes variés pour désigner un même trouble :
TDL, dysphasie, trouble primaire du langage, trouble du
langage, etc.
-Recherche de critères d'inclusion ou d'exclusion concer-
nant l'atteinte d'au moins deux composantes langagières, la
persistance des atteintes après six mois de suivi, la présence
d'un écart entre les capacités verbales et non verbales, l'éli-
mination obligatoire d'un TDAH ou d'un TSA avant de con-
clure. Utilisation du terme « retard de langage ».

-Établissement de niveaux de sévérité des atteintes selon les résultats obtenus à des tests standardisés

-Conceptualisation des troubles touchant la pragmatique ou la communication sociale comme étant partie intégrante du TDL ou d'un autre trouble (p. ex., TCS, TSA)

\section{Défi d'arrimage}

Non consensuel entre les professions, mésinterprétations possibles

Actualisé en neuropsychologie mais tombé en désuétude en orthophonie et reconnu instable par le DSM-5

Mésinterprétations et confusions possibles, enjeu d'accès aux services

Application par certains cliniciens de critères non soutenus scientifiquement, désuets ou administratifs (liés à l'accès aux services scolaires ou gouvernementaux)

Tests et barèmes employés variables selon les professionnels, confusion possible avec la sévérité des impacts fonctionnels, confusions avec critères administratifs d'accès aux services et aux subventions

Non consensuel entre les professions, confusion possible dans la compréhension des profils, enjeux d'accès aux services 
d'adaptation ou d'apprentissage. L'allocation budgétaire allouée à " l'élève handicapé par une déficience langagière " (identifié par le code 34) est ainsi basée sur des critères de sévérité des atteintes langagières (Gouvernement du Québec, 2007), critères désormais désuets qu'il faudra corriger le plus tôt possible.

Les professionnels impliqués doivent mettre à profit leurs expertises complémentaires tout en se concertant pour la protection du public. Ceci suppose de faire les références appropriées au bon moment, mais également de travailler en interdisciplinarité pour permettre une analyse globale des forces et des défis de la personne aux prises avec un TDL. Pour y arriver, il est nécessaire d'employer un langage compréhensible pour toutes les disciplines impliquées. Bien que l'adoption d'une terminologie commune comporte son lot de défis, il s'agit d'une étape essentielle pour mieux répondre aux besoins des individus ayant un TDL et pour favoriser leur intégration et leur participation sociales (OOAQ, 2018b).

\section{Références}

American Psychiatric Association [APA] (2015). DSM -5: manuel diagnostique et statistique des troubles mentaux, (5e éd.; traduit par M.-A. Crocq et J.D. Guelfi). Issy-les-Moulineaux, France: Elsevier Masson.

Aram, D. M., Morris, R., \& Hall, N. E. (1992). The validity of discrepancy criteria for identifying children with developmental language disorders. Journal of Learning Disabilities, 25, 549-554. doi: 10.1177/002221949202500902

Aram, D. M., Morris, R., \& Hall, N. E. (1993). Clinical and Research Congruence in Identifying Children With Specific Language Impairment. Journal of Speech, Language, and Hearing Research, 36, 580-591. doi:10.1044/jshr.3603.580

American Speech-Language-Hearing Association [ASHA]. (2016). Scope of practice in speechlanguage pathology. Repéré à www.asha.org/ policy/

American Speech-Language-Hearing Association [ASHA]. (2018). Kaleidoscopic Labels : The SLI/ DLD language disorder debate. The ASHA Leader, 23. Repéré à https://leader.pubs.asha.org/toc/ leader $/ 23 / 12$

Barnett, D. W., Neely, E., Wolsing, L., \& Bunger, C. E. (2006). Response to intervention for young children with extremely challenging behaviors: What it might look like. School Psychology Review, 35, 568-582.

Benner, G. J. (2005). Language skills of elementaryaged children with emotional and behavioral disorders. Great Plains Research, 15, 251-265.
Bishop, D. V. M. (2017). Why is it so hard to reach agreement on terminology? The case of developmental language disorder (DLD). International Journal of Language \& Communication Disorders, 52, 671-680. doi: 10.1111/1460-6984.12335

Bishop, D. V. M. \& Rutter, M. (2008). Neurodevelopmental disorders: conceptual issues. Rutter's child and adolescent psychiatry, 32-41.

Bishop, D. V. M. (2010). Which neurodevelopmental disorders get researched and why? PLoS One, 5, e15112. doi: 10.1371/journal.pone.0015112

Bishop, D. V. M. (2014). Ten questions about terminology for children with unexplained language problems. International Journal of Language \& Communication Disorders, 49, 381415. doi: 10.1111/1460-6984.12101

Bishop, D. V. M., Clark, B., Conti-Ramsden, G., Norbury, C. F., \& Snowling, M. J. (2012). RALLI: An internet campaign for raising awareness of language learning impairments. Child Language Teaching and Therapy, 28, 259-262. doi: 10.1177/0265659012459467

Bishop, D. V. M., Snowling, M. J., Thompson, P. A., Greenhalgh, T., \& CATALISE consortium. (2016). CATALISE: A Multinational and Multidisciplinary Delphi Consensus Study. Identifying Language Impairments in Children. PLoS One, 11, e0158753. doi:10.1371/journal.pone.0158753

Bishop, D. V. M., Snowling, M. J., Thompson, P. A., Greenhalgh, T., \& CATALISE-consortium. (2017). Phase 2 of CATALISE: a multinational and multidisciplinary Delphi consensus study of problems with language development: Terminology. Journal of Child Psychology and Psychiatry, 58, 1068-1080. doi: 10.1111/ jcpp. 12721

Botting, N. (2005). Non-verbal cognitive development and language impairment. Journal of Child Psychology and Psychiatry, 46, 317-326. doi:10.1111/j.1469-7610.2004.00355.x

Botting, N., Bean-Ellawadi, A., \& Williams, D. (2016). Language impairments in childhood - A range of profiles, a variety of reasons. Autism \& Developmental Language Impairments, 1, 1-2. doi:10.1177/2396941516654609

Braden, J. P. \& Weiss, L. (1988). Effects of simple difference versus regression discrepancy methods: An empiracal study. Journal of School Psychology, 26, 133-142.

Brown-Chidsey, R. \& Steege, M. W. (2011). Response to intervention: Principles and strategies for effective practice. New York, NY : Guilford Press.

Clegg, J., Hollis, C., Mawhood, L., \& Rutter, M. (2005). Developmental language disorders - a follow-up in later adult life. Cognitive, language and psychosocial outcomes. Journal of Child 
Psychology and Psychiatry, 46, 128-149. doi:10.1111/j.1469-7610.2004.00342.x

Cohen, N. J., Davine, M., \& Meloche-Kelly, M. (1989). Prevalence of unsuspected language disorders in a child psychiatric population. Journal of the American Academy of Child \& Adolescent Psychiatry, 28, 107-111.

Constantino, J., Todorov, A., Hilton, C., Law, P., Zhang, Y., Molloy, E., . . Geschwind, D. (2013). Autism recurrence in half siblings: strong support for genetic mechanisms of transmission in ASD. Molecular psychiatry, 18, 137-138.

Conti-Ramsden, G. \& Botting, N. (1999). Classification of children with specific language impairment: Longitudinal considerations. Journal of Speech, Language, and Hearing Research, 42, 1195-1204.

Conti-Ramsden, G. \& Botting, N. (2008). Emotional health in adolescents with and without a history of specific language impairment (SLI). Journal of Child Psychology and Psychiatry, 49, 516-525. doi:10.1111/j.1469-7610.2007.01858.x

Conti-Ramsden, G., Durkin, K., Toseeb, U., Botting, N., \& Pickles, A. (2018). Education and employment outcomes of young adults with a history of developmental language disorder. International Journal of Language \& Communication Disorders, 53, 237-255. doi:10.1111/1460-6984.12338

Conti-Ramsden, G., St Clair, M. C., Pickles, A., \& Durkin, K. (2012). Developmental trajectories of verbal and nonverbal skills in individuals with a history of specific language impairment: From childhood to adolescence. Journal of Speech, Language, and Hearing Research, 55, 1716-1735.

Croteau, C., Morin, C., Fournier, M., Le Dorze, G., Tessier, A., McIntyre, J., . . Choquette, V. (2015). Développement et validation d'un outil de mesureÉvaluation des facteurs environnementaux influençant la participation sociale des élèves du primaire présentant un trouble de la communication. Canadian Journal of SpeechLanguage Pathology \& Audiology, 39, 146-161.

Cunningham, B. J., Washington, K. N., Binns, A., Rolfe, K., Robertson, B., \& Rosenbaum, P. (2017). Current methods of evaluating speech-language outcomes for preschoolers with communication disorders: A scoping review using the ICF-CY. Journal of Speech, Language, and Hearing Research, 60, 447-464.

Durkin, K. \& Conti-Ramsden, G. (2010). Young people with specific language impairment: A review of social and emotional functioning in adolescence. Child Language Teaching and Therapy, 26, 105-121. doi: $10.1177 / 0265659010368750$
Elbro, C., Dalby, M., \& Maarbjerg, S. (2011). Language-learning impairments: a 30-year followup of language-impaired children with and without psychiatric, neurological and cognitive difficulties. International Journal of Language and Communication Disorder, 46, 437-448. doi:10.1111/j.1460-6984.2011.00004.x

Feeney, R., Desha, L., Khan, A., Ziviani, J., \& Nicholson, J. M. (2016). Speech and Language Difficulties Along with Other Child and Family Factors Associated with Health Related Quality of Life of Australian Children. Applied Research in Quality of Life, 11, 1379-1397. doi:10.1007/s11482 -015-9443-6

Flanagan, D. P. \& Alfonso, V. C. (2017). Essentials of WISC-V assessment. Hoboken, NJ: John Wiley \& Sons.

Fougeyrollas, P. (2010). La funambule, le fil et la toile: transformations réciproques $d u$ sens $d u$ handicap. Québec, Canada : Presses de l'Université Laval.

Fougeyrollas, P., Cloutier, R., Bergeron, H., Côté, J., \& St-Michel, G. (1998). Classification québécoise Processus de production du handicap. Québec, Canada : Réseau international sur le Processus de production du handicap.

Gérard, C.-L. (1993). L'enfant dysphasique. Bruxelles, Belgique : De Boeck Université.

Gernsbacher, M. A., Morson, E. M., \& Grace, E. J. (2016). Language and speech in autism. Annual review of linguistic, 2, 413-325. doi:10.1146/ annurev-linguist-030514-124824

Gillberg, C. (2010). The ESSENCE in child psychiatry: early symptomatic syndromes eliciting neurodevelopmental clinical examinations. Research in developmental disabilities, 31, 15431551.

Gillberg, C., Gillberg, I. C., Rasmussen, P., Kadesjö, B., Söderström, H., Råstam, M., . . . Niklasson, L. (2004). Co-existing disorders in ADHDimplications for diagnosis and intervention. European child \& adolescent psychiatry, 13, 80-92.

Gingras, M.-P. (2017, 23 juillet). Le nouveau « Trouble développemental du langage » : 57 experts se prononcent sur la terminologie entourant les troubles du langage [Billet de blogue]. Repéré à https://cuitdanslebec.wordpress.com/2017/07/23/lenouveau-trouble-du-langage-developpemental-57experts-se-prononcent-sur-la-terminologieentourant-les-troubles-du-langage/

Gomersall, T., Spencer, S., Basarir, H., Tsuchiya, A., Clegg, J., Sutton, A., \& Dickinson, K. (2015). Measuring quality of life in children with speech and language difficulties: a systematic review of existing approaches. International Journal of Language \& Communication Disorders, 50, 416435. doi:10.1111/1460-6984.12147 
Gooch, D., Hulme, C., Nash, H. M., \& Snowling, M. J. (2014). Comorbidities in preschool children at family risk of dyslexia. Journal of Child Psychology and Psychiatry, 55, 237-246.

Gouvernement du Québec. (2007). L'organisation des services éducatifs aux élèves à risque et aux élèves handicapés ou en difficulté d'adaptation ou d'apprentissage (EHDAA). Québec: Gouvernement $\mathrm{du}$ Québec. Repéré à http:// www.education.gouv.qc.ca/fileadmin/site_web/ documents/dpse/adaptation_serv_compl/19. 7065.pdf

Heffer, R. W., Barry, T. D., \& Garland, B. H. (2009). History, overview, and trends in child and adolescent psychological assessment. Dans J. L. Matson, F. Andrasik, \& M. L. Matson (Eds.), Assessing childhood psychopathology and developmental disabilities (pp. 3-29). New York, NY: Springer.

Hill, E. L. (2001). Non-specific nature of specific language impairment: a review of the literature with regard to concomitant motor impairments. International Journal of Language \& Communication Disorders, 36, 149-171.

Hollo, A., Wehby, J. H., \& Oliver, R. M. (2014). Unidentified language deficits in children with emotional and behavioral disorders: A metaanalysis. Exceptional Children, 80, 169-186. doi: $10.1177 / 001440291408000203$

Hsu, C.-C. \& Sandford, B. A. (2007). The Delphi technique: making sense of consensus. Practical assessment, research \& evaluation, 12, 1-8.

Jacobs, F. H. (2017). The five-tiered approach to evaluation: Context and implementation. Dans H. B. Weiss \& F. H. Jacobs (2017) Evaluating family programs: Current issues in theory and policy ( $\mathrm{p}$. 37-68). New York, NY: Routledge.

Johnson, C. J., Beitchman, J. H., \& Brownlie, E. (2010). Twenty-year follow-up of children with and without speech-language impairments: Family, educational, occupational, and quality of life outcomes. American journal of speech-language pathology, 19, 51-65. doi: 10.1044/1058-0360 (2009/08-0083)

Kwok, E. Y., Brown, H. M., Smyth, R. E., \& Cardy, J. O. (2015). Meta-analysis of receptive and expressive language skills in autism spectrum disorder. Research in Autism Spectrum Disorders, 9, 202-222.

Law, J., Reilly, S., \& Snow, P. C. (2013). Child speech, language and communication need reexamined in a public health context: a new direction for the speech and language therapy profession. International Journal of Language \& Communication Disorders, 48, 486-496. doi: 10.1111/1460-6984.12027
Lévesque, A.-I. (2017). La réponse à l'intervention [Dossier thématique]. Repéré à http://rire.ctreq.qc.ca/2017/11/rai-dt/.

Leyfer, O. T., Tager-Flusberg, H., Dowd, M., Tomblin, J. B., \& Folstein, S. E. (2008). Overlap between autism and specific language impairment: comparison of autism diagnostic interview and autism diagnostic observation schedule scores. Autism Research, 1, 284-296.

Lussier, F., Chevrier, E., \& Gascon, L. (2018). Neuropsychologie de l'enfant-3e éd.: Troubles développementaux et de l'apprentissage. Malakoff, France: Dunod.

Lyons, R., Byrne, M., Corry, T., Lalor, L., Ruane, H., Shanahan, R., \& McGinty, C. (2008). An examination of how speech and language therapists assess and diagnose children with specific language impairment in Ireland. Internationnal Journal of Speech-Language Pathology, 10, 425-437. doi:10.1080/17549500802422569

Maillart, C. (2018). Le projet CATALISE, phase 2 « Terminologie ». Impacts sur la nomenclature des prestations de logopédie en Belgique. UPLF-Info, 35, 4-17.

Mash, E. J. \& Barkley, R. A. (2014). Child psychopathology. New York, NY: Guilford Publications.

McLeod, S. \& Harrison, L. J. (2009). Epidemiology of speech and language impairment in a nationally representative sample of 4-to 5-year-old children. Journal of Speech, Language, and Hearing Research, 52, 1213-1229.

McLeod, S. \& Threats, T. T. (2009). The ICF-CY and children with communication disabilities. International Journal of Speech-Language Pathology, 10, 92-109. doi: 10.1080/17549500701834690

Miller, C. A. \& Gilbert, E. (2008). Comparison of performance on two nonverbal intelligence tests by adolescents with and without language impairment. Journal of Communication Disorders, 41, 358-371.

Norbury, C. F., Gooch, D., Wray, C., Baird, G., Charman, T., Simonoff, E., . . . Pickles, A. (2016). The impact of nonverbal ability on prevalence and clinical presentation of language disorder: evidence from a population study. Journal of Child Psychology and Psychiatry, 57, 1247-1257. doi: $10.1111 /$ jepp. 12573

Ordre des orthophonistes et audiologistes du Québec. (2018a). Fiche d'avancement de la pratique: Trouble développemental du langage. Repéré à http://www.ooaq.qc.ca/Envoi_aux_membres/2018documents/Trouble $\% 20$ developmental $\% 20 \mathrm{du} \%$ 20langage_mai\%202018.pdf?

tm_source=Liste+Avec+Promo\&utm_campaign=f5 1a $\overline{6}$ b1092-EMAIL_CAMPAIGN_2017_09_12\&ut 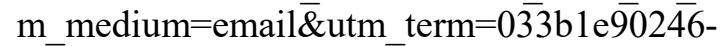 f51 a6b1092-146698065 
Ordre des orthophonistes et audiologistes du Québec. (2018b). Trouble développemental du langage (TDL) - Fiche explicative pour les professionnels. Dans Ordre des orthophonistes et audiologistes du Québec. Repéré à http://www.ooaq.qc.ca/ orthophonie/index.html

Organisation mondiale de la santé [OMS]. (2007). Classification internationale du fonctionnement, $d u$ handicap et de la santé : version pour enfants et adolescents (CIF-EA). Genève, Suisse: OMS.

Parsons, S., Schoon, I., Rush, R., \& Law, J. (2011). Long-term outcomes for children with early language problems: Beating the odds. Children \& Society, 25, 202-214.

Rapin, I. \& Allen, D. (1983). Developmental language disorders: Nosologic considerations. Neuropsychology of Language, Reading, and Spelling, 4, 155-184.

Reilly, S., Bishop, D. V. M., \& Tomblin, B. (2014). Terminological debate over language impairment in children: forward movement and sticking points. International Journal of Language \& Communication Disorders, 49, 452-462. doi: 10.11 11/1460-6984.12111

Reilly, S., Tomblin, B., Law, J., McKean, C., Mensah, F. K., Morgan, A., . . . Wake, M. (2014). Specific language impairment: a convenient label for whom? International Journal of Language and Communication Disorder, 49, 416-451. doi: 10.1111/1460-6984.12102

Réseau international sur le Processus de production du handicap. (2019). Repéré https://ripph.qc.ca

Silva, P. A. (1980). The Prevalence, Stability and Significance of Developmental Language Delay in Preschool Children. Developmental Medicine \& Child Neurology, 22, 768-777. doi: 10.1111/j.14698749.1980.tb03743.x

Smolla, N., Béliveau, M.-J., Noël, R., Breault, C., Lévesque, A., Berthiaume, C., \& Martin, V. (2015). La pertinence de l'inquiétude parentale pour le développement langagier du jeune enfant référé en psychiatrie. Revue québécoise de psychologie, 36, 235-263.

Snowling, M. J., Duff, F. J., Nash, H. M., \& Hulme, C. (2016). Language profiles and literacy outcomes of children with resolving, emerging, or persisting language impairments. Journal of Child Psychology and Psychiatry, 57, 1360-1369.

Spaulding, T. J., Plante, E., \& Farinella, K. A. (2006). Eligibility criteria for language impairment: is the low end of normal always appropriate? Language, Speech, and Hearing Services in Schools, 37, 61-72.

St Clair, M. C., Pickles, A., Durkin, K., \& ContiRamsden, G. (2011). A longitudinal study of behavioral, emotional and social difficulties in individuals with a history of specific language impairment (SLI). Journal of Communication
Disorders, 44, 186-199. doi: 10.1016/ j.jcomdis.2010.09.004

Sylvestre, A., Brisson, J., Lepage, C., Nadeau, L., \& Deaudelin, I. (2016). Social participation of children age 8-12 with SLI. Disability and Rehabilitation, 38, 1146-1156. doi: 10.310 9/09638288.2015.1074730

Tessier, A. \& Valade, S. (2017). Organisation $d u$ continuum et de la dispensation des services aux enfants âgés de 2 à 9 ans présentant un trouble développemental $d u$ langage (trouble primaire $d u$ langage). Repéré à https://www.inesss.qc.ca/nc/ publications/publications/publication/organisationdu-continuum-et-de-la-dispensation-des-servicesaux-enfants-ages-de-2-a-9-ans-presentan.html

Thomas-Stonell, N., Washington, K., Oddson, B., Robertson, B., \& Rosenbaum, P. (2013). Measuring communicative participation using the FOCUS(C): Focus on the Outcomes of Communication Under Six. Child, 39, 474-480. doi: 10.1111/cch.12049

Thordardottir, E., Kehayia, E., Lessard, N., Sutton, A., \& Trudeau, N. (2010). Typical performance on tests of language knowledge and language processing of French-speaking 5-year-olds. Revue canadienne d'orthophonie et d'audiologie, 34, 5-16.

Thordardottir, E., Kehayia, E., Mazer, B., Lessard, N., Majnemer, A., Sutton, A., . . . Chilingaryan, G. (2011). Sensitivity and Specificity of French Language and Processing Measures for the Identification of Primary Language Impairment at Age 5. Journal of Speech Language and Hearing Research, 54. doi: 10.1044/1092-4388(2010/090196)

Tomblin, J. B., Records, N. L., Buckwalter, P., Zhang, X., Smith, E., \& O'Brien, M. (1997). Prevalence of Specific Language Impairment in Kindergarten Children. Journal of Speech, Language, and Hearing Research, 40, 1245-1260. doi: 10.1044/ jslhr.4006.1245

Toseeb, U., Pickles, A., Durkin, K., Botting, N., \& Conti -Ramsden, G. (2017). Prosociality from early adolescence to young adulthood: A longitudinal study of individuals with a history of language impairment. Research in Developmental Disabilities, $62,148-159$.

Volkers, N. (2018, December). Diverging views on language disorders. The ASHA Leader, 23, 44-53.

Washington, K. N. (2009). Using the ICF within speechlanguage pathology: Application to developmental language impairment. Advances in Speech Language Pathology, 9, 242-255. doi: 10.1080/14417040701261 525

Reçu le 23 novembre 2018 Révision reçue 18 avril 2019 Accepté le 17 juillet 2019 